\title{
Mechanical Properties of Flexible Polypyrrole-Based Conducting Polymer Alloy Films
}

\author{
Osamu NiwA, Masami KAKUCHI, and Toshiaki TAMAMURA \\ NTT Electrical Communications Laboratories, Tokai, Ibaraki 319-11, Japan
}

(Received April 20, 1987)

\begin{abstract}
The electrochemical polymerization of pyrrole on an electrode covered with an insulating polymer film,produces conducting polymer alloy films of polypyrrole (PPy) and insulating polymer. By using vinylidene fluoride-trifluoroethylene copolymer (P(VDF-TrFE)) as an insulating polymer, very flexible and stretchable conducting films were obtained. The mechanical properties of the alloy films were studied by measuring tensile strength and dynamic modulus of elasticity. The excellent mechanical properties of $\mathrm{P}(\mathrm{VDF}-\mathrm{TrFE})$ did not change by the incorporation of PPy up to a charge density of $0.5 \mathrm{C} \mathrm{cm}^{-2}$. A further increase of charge density caused a reduction of dynamic moduli of the alloy films. The alloy films with the charge density of $0.5 \mathrm{C} \mathrm{cm}^{-2}$ extended more than $100 \%$ and their surface resistance was almost constant up to $50 \%$ elongation. The elongation more than $60 \%$ resulted in a large increase of surface resistance, because inhomogeneous fracture occurs from the electrode side of the film. A comparison of the mechanical properties between P(VDF-TrFE)-PPy and other alloy films based on poly(vinyl chloride) were also studied.
\end{abstract}

KEY WORDS Electrochemical Polymerization / Mechanical Property /

Polypyrrole / Poly(vinyl chloride) / Vinylidene Fluoride-Trifluoroethylene

Copolymer /

Various aromatic compounds have been found to electrochemically polymerize, forming electrical conducting polymers on the electrode. ${ }^{1}$ Polypyrrole (PPy) is one of the representative conducting polymers with performance of a high conductivity $c a .100 \mathrm{~S} \mathrm{~cm}^{-1}$ and good film forming properties. ${ }^{2}$ Various applications such as antistatic plastic films, rechargeable batteries and ion separation membranes have been proposed. However, for the practical use of PPy significant improvement of mechanical property and processability is required. PPy films obtained by electrochemical polymerization are almost insoluble, brittle and cannot extend more than $5 \%{ }^{3}$ Many efforts have concentrated on the improvement of the film quality of PPy. Iwata $e t$ al. ${ }^{4}$ obtained the flexible pure PPy with high conductivity more than $300 \mathrm{Scm}^{-1}$ by poly- merizing pyrrole at a low temperature about $-30^{\circ} \mathrm{C}$.

The most attractive feature of this electrochemical polymerization is the capability of forming various hybrid conducting materials with different types of polymers. By using polymeric anions as electrolyte, PPy-polymer anion complexes can be easily prepared, and flexible polymeric anions can improve the mechanical properties of PPy. Bates et al. ${ }^{5}$ reported that PPy-sulphonated styrene(hydrogenated) butadiene tri-block copolymer complex becomes flexible and can be extended up to $400 \%$. However, the electrical conductivity of PPy-polyanion becomes less than $10^{-2} \mathrm{~S} \mathrm{~cm}^{-1}$, which is lower by 4 orders of magnitude compared with PPy single film.

Another type of hybrid material can be prepared by the electrochemical polymer- 

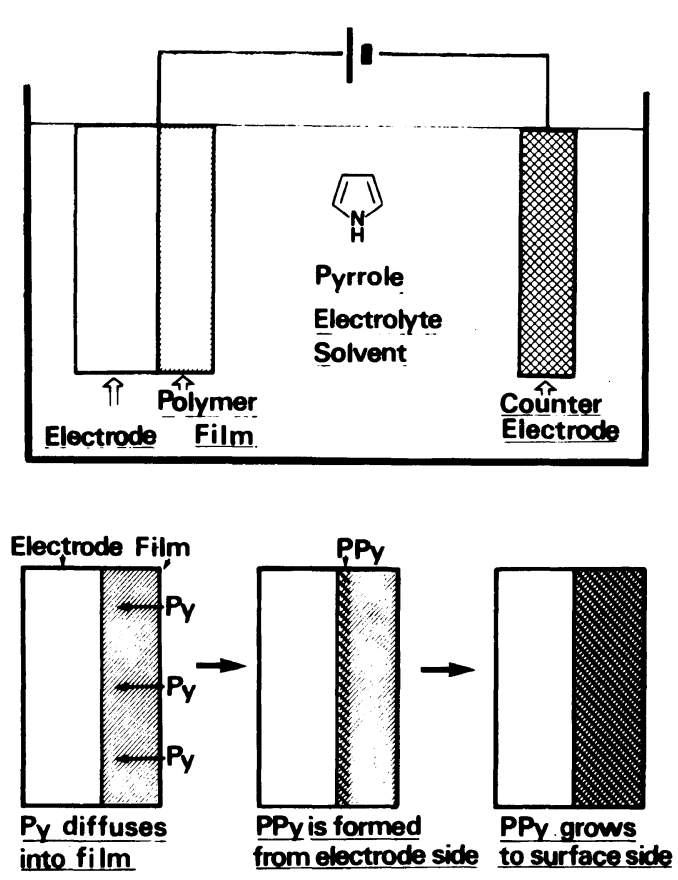

Figure 1. Schematic procedure for the preparation of PPy-based polymer alloy films.

ization of pyrrole on an electrode whose surface is covered with ordinary insulating polymer film. ${ }^{6,7}$ The schematics of this process are shown in Figure 1. Pyrrole molecules and electrolyte anions diffuse into the insulating film and start to polymerize between the electrode and polymer film. Highly conducting PPy grows inside the polymer film from the electrode surface and makes the whole of the film conductive. This method is very useful to control the physical properties of PPy such as electrical conductivity, ${ }^{8,9}$ optical transmittance $^{10}$ of PPy and has great potential to improve the mechanical properties and processability of conducting polymers. The mechanical properties of alloy films were improved when PPy was incorporated into a poly(vinyl chloride) (PVC)-polychloroprene blend, and their elongation at break $\left(\gamma_{\mathrm{B}}\right)$ increased up to $30 \%{ }^{11}$

We studied the dependence of the mechanical properties of the alloy films on polymer species, and found that vinylidene fluoride- trifluoroethylene copolymer ( $\mathrm{P}(\mathrm{VDF}-\mathrm{TrFE}))$ is suitable to increase the mechanical properties of PPy, particularly its ductility. In this paper, the mechanical properties of $\mathrm{P}(\mathrm{VDF}-$ TrFE)-PPy alloy films are studied and compared with the alloy films prepared using poly(vinyl chloride) (PVC), ethylene-vinyl acetate-vinyl chloride copolymer (P(EVA-VC)) which are representative of amorphous polymers.

\section{EXPERIMENTAL}

$\mathrm{P}(\mathrm{VDF}-\mathrm{TrFE}), \mathrm{PVC}$, and P(EVA-VC)) were obtained from Daikin Kogyo, Wako Chemicals and Nippon Zeon, respectively, and their properties and composition are shown in Table I. Each polymer was coated on an indium-tin-oxide (ITO) glass substrate by a casting method. Film thickness was kept at $20 \mu \mathrm{m}$. The ITO substrate with the polymer film was immersed in an acetonitrile-based solution containing $1 \mathrm{moll}^{-1}$ pyrrole and $0.3 \mathrm{moll}^{-1}$ of tetraethylammonium $p$-toluenesulfonate (PTSN) as a suppporting electrolyte. The electrochemical polymerization of pyrrole was carried out by applying a constant voltage of $2.0 \mathrm{~V}$ between the ITO electrode and a Pt coated Ti mesh as a counterelectrode. The amount of PPy incorporated into the film was evaluated by measuring the charge density with a digital coulomb meter (PAR-379). The yield of PPy is almost proportional to the charge used for the polymerization of pyrrole. $0.58 \mathrm{mg}$ of PPy was obtained with a charge density of $1.0 \mathrm{C}$. This value is about $7 \%$ larger than the calculated value of PPy-PTSN with the same amount of charge, but gradually decreases and approaches the calculated value by vacuum drying. After the polymerization, the alloy film was washed with fresh acetonitrile or ethanol and stripped from the electrode. The surface resistances $\left(R_{\mathrm{s}}\right)$ of the alloy films was evaluated from both the surface and electrode side with a four terminal method. The mechanical properties of the 
Table I. The physical properties and composition of base films

\begin{tabular}{|c|c|c|c|c|}
\hline \multirow{2}{*}{ Polymers } & Composition & & $T_{\mathrm{g}}$ & \multirow{2}{*}{ Morphology } \\
\hline & $w t \%$ & & ${ }^{\circ} \mathrm{C}$ & \\
\hline \multirow[t]{2}{*}{$P(V D F-T r F E)$} & Vinylydene fluoride & 52.5 & -35 & Crystalline \\
\hline & Trifluoroethylene & 47.5 & & \\
\hline PVC & Homopolymer & & 85 & Amorphous \\
\hline \multirow[t]{3}{*}{$\mathrm{P}(\mathrm{EVA}-\mathrm{VC})$} & Ethylene & 50.6 & -60 & Amorphous \\
\hline & Vinyl acetate & 15.1 & & \\
\hline & Vinyl chloride & 34.3 & & \\
\hline
\end{tabular}

Table II. Results of surface resistance and stress-strain test of PVC/PPy, P(EVA-VC)/PPy, and $\mathrm{P}(\mathrm{VDF}-\mathrm{TrFE}) / \mathrm{PPy}$ compared with their base films. Films constant about $6 \%$ PPy by polymerization with a charge density of $0.5 \mathrm{Ccm}^{-2}$

\begin{tabular}{|c|c|c|c|}
\hline \multirow{2}{*}{ Polymers } & Surface resistance & Tensile strength & \multirow{2}{*}{$\begin{array}{c}\text { Elongation at } \\
\text { break } / \%\end{array}$} \\
\hline & $\Omega$ & $\mathrm{MPa}$ & \\
\hline PPy & 8 & 61 & 4 \\
\hline $\mathrm{P}(\mathrm{VDF}-\mathrm{TrFE})$ & & 17 & 600 \\
\hline $\mathrm{P}(\mathrm{VDF}-\mathrm{TrFE}) / \mathrm{PPy}$ & 223 & 18 & 122 \\
\hline PVC & & 39 & 120 \\
\hline PVC/PPy & 360 & 43 & 39 \\
\hline P(EVA-VC) & & 12 & 290 \\
\hline $\mathrm{P}(\mathrm{EVA}-\mathrm{VC}) / \mathrm{PPy}$ & 136 & 44 & 18 \\
\hline
\end{tabular}

alloy film were investigated from the measurement of the stress-strain test and dynamic modulus of elasticity using the Strograph $T$ (Toyo Seiki) and Reovibron DDV-IIIA (Toyo Baldwin), respectively. The film morphology was studied by scanning electron microscope (SEM JEOL JSM-840) observation.

\section{RESULTS AND DISCUSSION}

\section{Stress-Strain Test}

Table II shows the mechanical properties of PVC-PPy, P(EVA-VC)-PPy, and P(VDFTrFE)-PPy polymer alloy films compared with their base films and PPy single film. P(VDFTrFE), which is known to be ferroelectric, flexible and a crystalline polymer with a very low $T_{\mathrm{g}}$. On the other hand, PVC is relatively hard and almost an amorphous polymer containing micro-crystalline region with $T_{\mathrm{g}}$ of $c a$. $80^{\circ} \mathrm{C}$. $\mathrm{P}(\mathrm{EVA}-\mathrm{VC})$ is a block copolymer of ethylene-vinyl acetate copolymer and vinyl chloride, and shows intermediate properties between PVC and P(VDF-TrFE), it is an amorphous polymer with very low $T_{\mathrm{g}}$.

Figures 2, 3, and 4 show the stress-strain curves of the alloy and pure films. PPy polymerized in each film with a charge density of $0.5 \mathrm{C} \mathrm{cm}^{-2}$. PPy single films showed a large tensile strength of $64 \mathrm{MPa}$, and small elongation at break $\left(\gamma_{\mathrm{B}}\right)$ less than $5 \%$. The stressstrain curve of PPy film is similar to aromatic polymers such as polystyrene and polyimide.

When PPy was incorporated into a polymer 


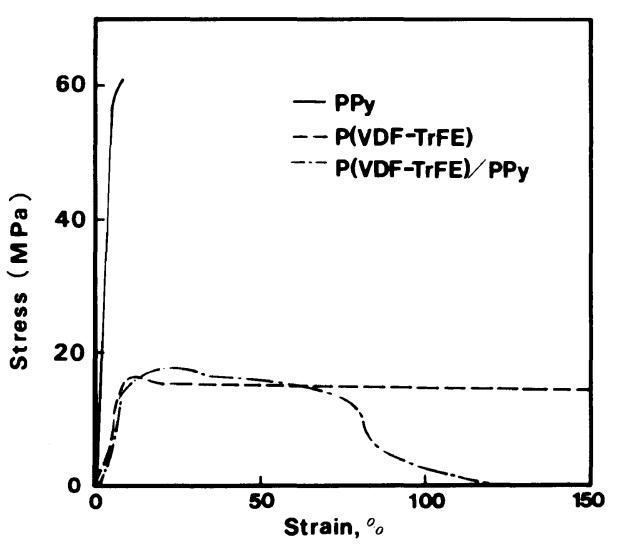

Figure 2. Stress-strain curves of PPy, P(VDF-TrFE), and P(VDF-TrFE)-PPy films.

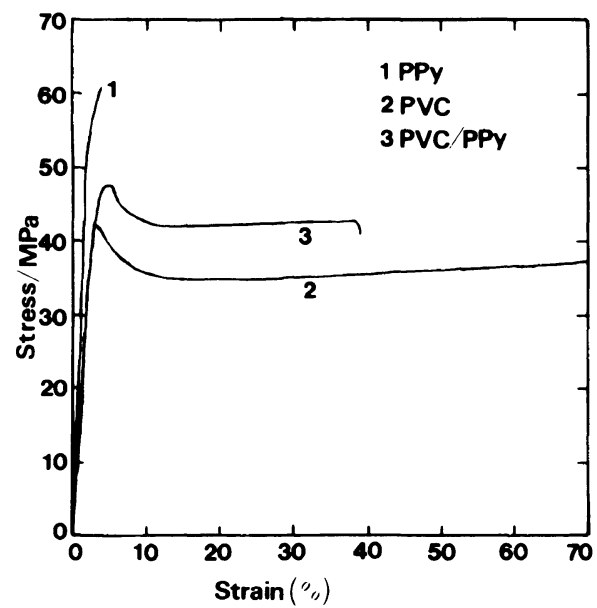

Figure 3. Stress-strain curves of PPy, PVC, and PVCPPy films.

film, the $\gamma_{\mathbf{B}}$ of each polymer film decreased, the degree varying with the base polymer. The P(VDF-TrFE)-PPy alloy film is very soft and flexible due to inheriting the properties of the base polymer which can be extended by more than $600 \%$. P(VDF-TrFE)-PPy alloy film showed a $\gamma_{B}$ more than $100 \%$. PVC-PPy alloy film showed $33 \% \gamma_{B}$, which is 3 times lower compared with PVC single film. Surprisingly, even through P(EVA-VC) film is soft and can be extended about $290 \%$, the $\gamma_{\mathrm{B}}$ of P(EVAVC)-PPy was only $20 \% \cdot \gamma_{B}$ of pure P(EVAVC) films was comparable to that of P(VDF-

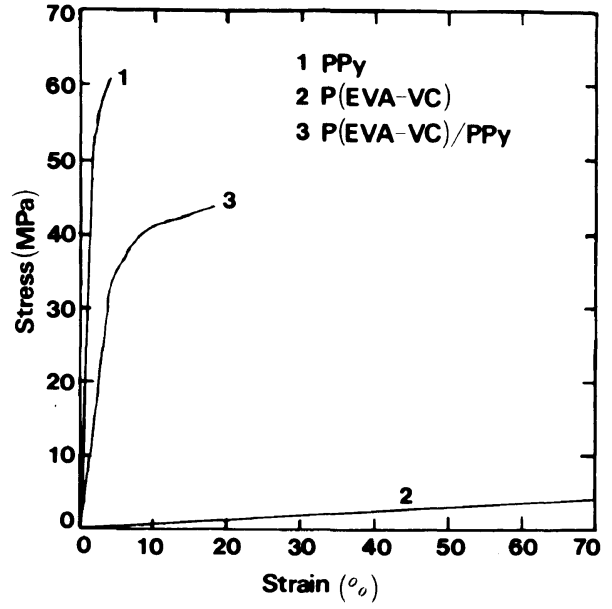

Figure 4. Stress-strain curves of PPy, P(EVA-VC), and P(EVA-VC)-PPy films.

TrFE), but the $\gamma_{\mathbf{B}}$ of P(EVA-VC)-PPy was 5 times lower than that of P(VDF-TrFE)-PPy. In the cases of P(VDF-TrFE)-PPy and PVCPPy alloy films, the shape of the stress-strain curves was similar to that of their base polymers at the early stage of elongation, and their tensile strength was almost the same as their base polymers. On the other hand, the stress-strain curve of $\mathrm{P}(\mathrm{EVA}-\mathrm{VC})-\mathrm{PPy}$ resembles pure PPy rather than P(EVA-VC) film, and its tensile strengh was much larger than that of the base polymer. These results indicate that the mechanical properties of the PPy based alloy films are not always reflected by the mechanical properties of their base films.

The difference of the mechanical properties between P(VDF-TrFE)-PPy and P(EVAVC)-PPy may be considered as follows. Since the molecular motion of host polymer chains are so rapid at room temperature due to very low $T_{g}$, both films can be easily extended. However, the elongation mechanism is clearly different between $\mathrm{P}(\mathrm{VDF}-\mathrm{TrFE})$ and $\mathrm{P}(\mathrm{EVA}-$ VC). $P(V D F-T r F E)$ film yields to a small strain, and thus the stress can be relaxed, because the rearrengement and orientation of $\mathrm{P}(\mathrm{VDF}-\mathrm{TrFE})$ chains easily occur as in the 
case of linear polyethylene. On the other hand, the stress-strain curves of the P(EVA-VC) film resembles the curve of typical elastomer. P(EVA-VC) film shows no yield point and its strain can be recovered when stress is removed. P(EVA-VC) chains cannot orient because molecular chains are fixed to each other. This type of film is very stretchable, but often breaks by a small scratch.

When PPy was incorporated into both films, the resultant films became harder because of the reduction of the molecular motion of host polymer main chains. P(VDF-TrFE)-PPy film can relax stress by the high degree of main chain orientation and prevent the development of cracks in the alloy film. On the other hand, P(EVA-VC)-PPy films cannot relax stress because of the absence of a suitable molecular rearrengement. The stress of P(EVA-VC)-PPy increases with a small strain and developes cracks which results in film breakage. In conclusion, linear chain crystalline polymers such as $\mathrm{P}(\mathrm{VDF}-\mathrm{TrFE})$ whose $T_{\mathrm{g}}$ is below the room temperature are the most suitable base films to prepare flexible conducting films by this electrochemical technique.

\section{Surface Resistance $\left(R_{\mathrm{s}}\right)$ Change during Elon- gation}

From the stress-strain test, it was confirmed that P(VDF-TrFE)-PPy alloy films show a high $\gamma_{\mathbf{B}}$. After the release of stress, P(VDFTrFE)-PPy alloy film suffers from film deformation. This means that the film structure changes by elongation, which could affect the electrical conductivity of the film. Then, the resistance change during elongation was studied with respect to film structure.

Figure 5 shows the $R_{\mathrm{s}}$ change of $\mathrm{P}\left(\mathrm{VDF}_{-}\right.$ TrFE)-PPy film prepared under the condition of $2 \mathrm{~V}, 0.5 \mathrm{Ccm}^{-2}$ accompanied by elongation. This film showed a conductivity in both film sides, indicating PPy chains to grow up to the film surface. However, the $R_{\mathrm{s}}$ values measured from the surface side varied from sample to sample, perhaps due to slight in-

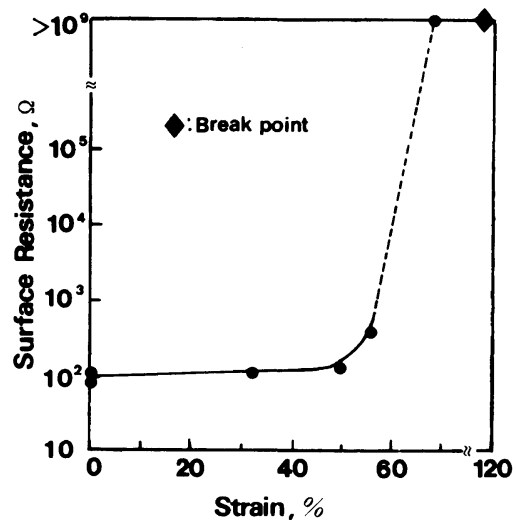

Figure 5. Surface resistance change of $P(V D F-T r F E)-$ PPy alloy film prepared under the condition of $2 \mathrm{~V}, 0.5$ $\mathrm{Ccm}^{-2}$ accompanied by elongation.

homogeneous growth of PPy toward the film surface, $R_{\mathrm{s}}$ was thus evaluated by using the electrode side resistance. P(VDF-TrFE)-PPy alloy film which $R_{\mathrm{s}}$ of an $300 \Omega$ did not change up to $50 \%$ elongation. This means that P(VDF-TrFE)-PPy film can be extended about 10 times higher than PPy single film without significant increase of $R_{\mathrm{s}}$. The flexible P(VDF-TrFE) layer may prevent fracture of the conducting path of PPy in the alloy film by relaxing the stress. However, $R_{\mathrm{s}}$ of $\mathrm{P}(\mathrm{VDF}-$ TrFE)-PPy alloy film rapidly increased by elongation more than $50 \%$, and became almost insulating at $60 \%$ elongation. The alloy film itself stood up to a $\gamma_{\mathrm{B}}$ of $120 \%$.

To study the fracture mechanisms of P(VDF-TrFE)-PPy films, the electrode side of P(VDF-TrFE)-PPy films was observed with SEM, and the results at $0,8,50$, and $64 \%$ elongation are shown in Figures $6 \mathrm{a}-\mathrm{d}$. At $8 \%$ elongation (Figure $6 \mathrm{~b}$ ), the electrode side was the almost same as the alloy film before elongation (Figure $6 \mathrm{a}, 0 \%$ elongation). At $50 \%$ elongation (Figure 6c), P(VDF-TrFE) PPy alloy films were highly oriented without crack generation. At $64 \%$ elongation (Figure $6 \mathrm{~d})$, the P(VDF-TrFE)-PPy layer near the electrode fractured, and a highly oriented layer was seen through the cracks. In a low magnification picture of Figure 6d many 


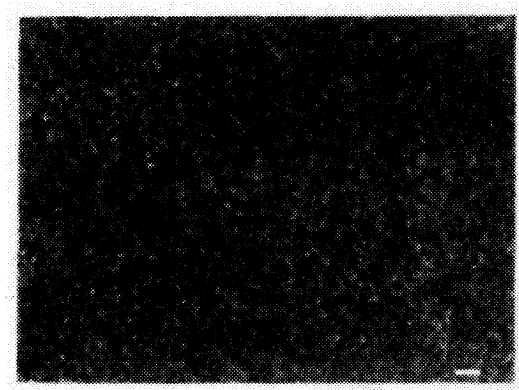

a

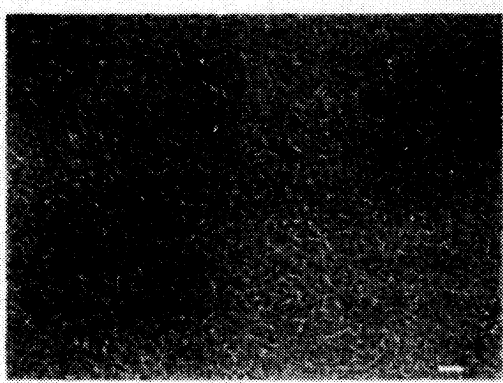

b

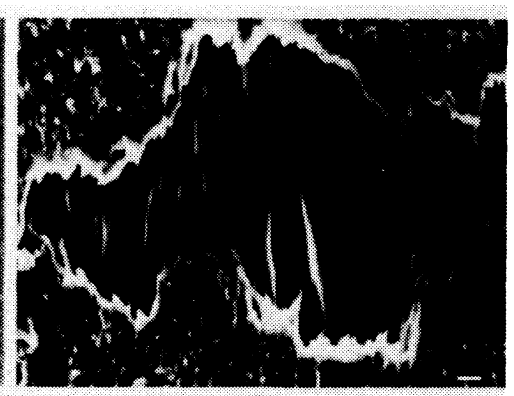

d.

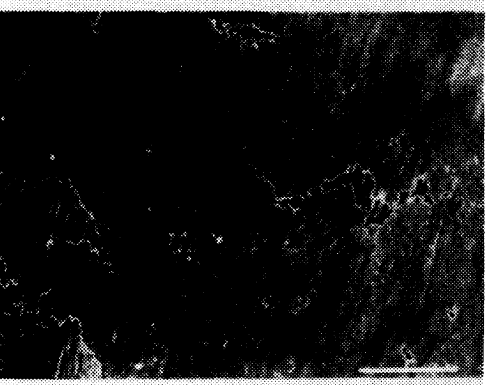

e

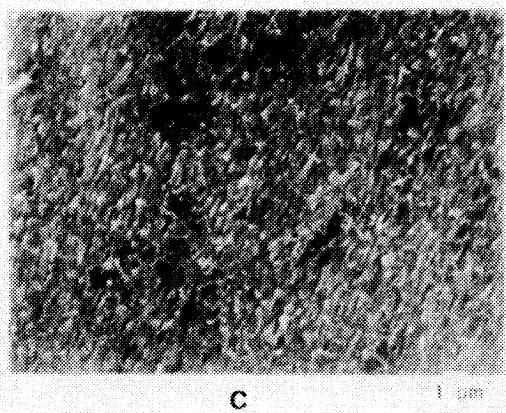

Figure 6. SEM pictures of the electrode side of P(VDF-TrFE)-PPy alloy films at 0, 80, 50, and 64\% elongation: a, $0 \%$; b, $8 \%$; c, 50\%; d, 64\%; e, 64\% (low magnification).

cracks developed on the electrode side (Figure 6e), and the layer near the electrode side was almost destroyed.

In a previous study on the morphology of PPy-based conducting alloy films, ${ }^{9}$ it was clarified that the mixing of PPy has a large distribution in the thickness direction. P(VDFTrFE)-PPy alloy film consists of three layers, as shown in Figure 7. The bottom layer contacting with the ITO electrode is a very thin PPy homopolymer film and the middle layer is a finely mixed layer of $\mathrm{P}(\mathrm{VDF}-\mathrm{TrFE})$ and PPy. This layer has a network-like PPy

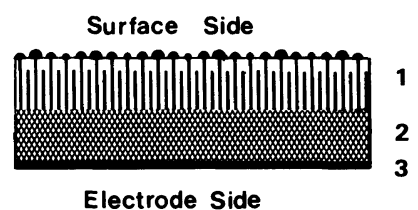

Figure 7. Schematic representation of the structure of alloy films: 1 , coarsely mixed layer; 2 , finely mixed layer; 3, pure PPy layer.

structure. The upper layer is mainly $\mathrm{P}(\mathrm{VDF}-$ TrFE) film in which relatively large PPy chains grow coarsely. The bottom 2 layers contribute to the conductivity in the electrode side of the film. Electrical conduction in the surface side 
was attained not within the surface side, but through the thickness direction along the coarsely mixed PPy chains.

When the stress is applied to this film, cracks may generate at first in the bottom pure PPy layer, but at this stage almost no decrease in electrical conductivity may be observed, because this layer is very thin and the middle layer is conductive. Further application of stress by elongation may develop cracks in the middle layer and the film becomes insulating when the cracks have completely broken the bottom 2 layers. This point corresponds to a $60 \%$ elongation, and beyond this point, the mechanical strength of the alloy film may totally depend on the upper film which is composed of mainly stretchable $\mathrm{P}(\mathrm{VDF}-$ TrFE). Unlike pure P(VDF-TrFE), this upper layer broke at $120 \%$ elongation. This film break may be caused by stress development from the coarsely distributed PPy chains.

Figure 8 shows the $R_{\mathrm{s}}$ change by elongation for PVC-PPy alloy film prepared at $2 \mathrm{~V}$, $0.5 \mathrm{C} \mathrm{cm}^{-2}$. PVC-PPy films became insulating when the films were stretched by more than $30 \%$, then the films soon broke at $40 \%$ elongation. In this case, the bottom 2 layers contributing to the conductivity in the electrode side broke at about $30 \%$ elongation, and cracks formed in these layers may rapidly develop in hard PVC film in a coarsely mixed upper layer.

\section{Dynamic Mechanical Property}

In order to get further information on the mechanical properties of $\mathrm{P}(\mathrm{VDF}-\mathrm{TrFE})-\mathrm{PPy}$ alloy films, dynamic and loss moduli were measured. However, no distinctive difference was observed by the incorporation of PPy up to a charge density of $0.5 \mathrm{Ccm}^{-2}$. Then, the films with charge densities of 1.0 , and $2.5 \mathrm{C} \mathrm{cm}^{-2}$ were subjected to the dynamic mechanical property measurements.

Figure 9 shows the temperature dependence of dynamic moduli of elasticity $(E)$ and loss moduli $(\tan \delta)$ for P(VDF-TrFE)-PPy alloy

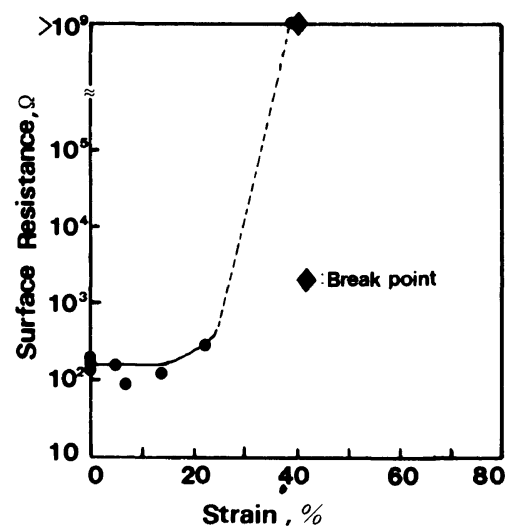

Figure 8. Surface resistance change of PVC-PPy alloy film prepared under the condition of $2 \mathrm{~V}, 0.5 \mathrm{C} \mathrm{cm}^{-2}$ accompanied by elongation.

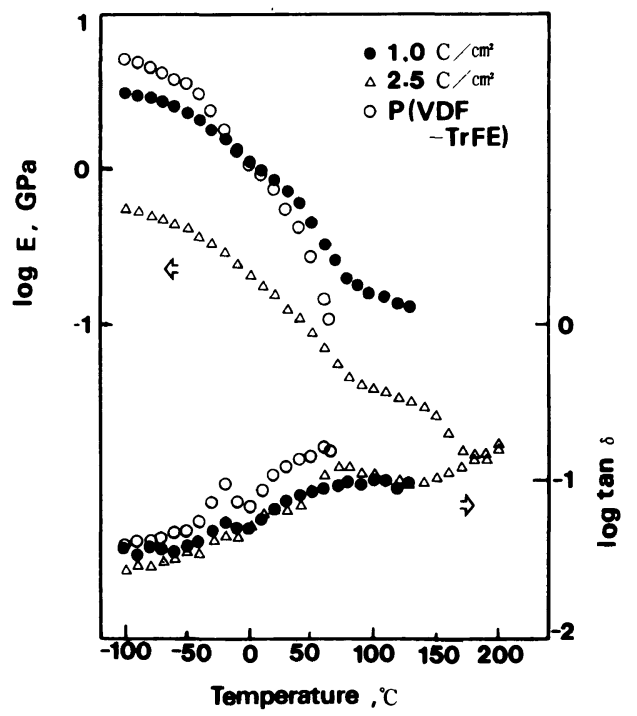

Figure 9. Temperature dependence of dynamic moduli of elasticity and loss moduli $(\tan \delta)$ for P(VDF-TrFE)PPy alloy films.

films. $E$ of $\mathrm{P}(\mathrm{VDF}-\mathrm{TrFE})-\mathrm{PPy}$ films became lower with increasing PPy content in the base film, and reduced by one order of magnitude with the charge density of $2.5 \mathrm{C} \mathrm{cm}^{-2}$. This is unusual in the case of an ordinary composite of two polymers, because PPy has a high $E$ larger than $1 \mathrm{GPa}$. At the early stage of polymerization, $\mathrm{P}(\mathrm{VDF}-\mathrm{TrFE})-\mathrm{PPy}$ film shows similar $E$ value to pure $\mathrm{P}(\mathrm{VDF}-\mathrm{TrFE})$ film, 


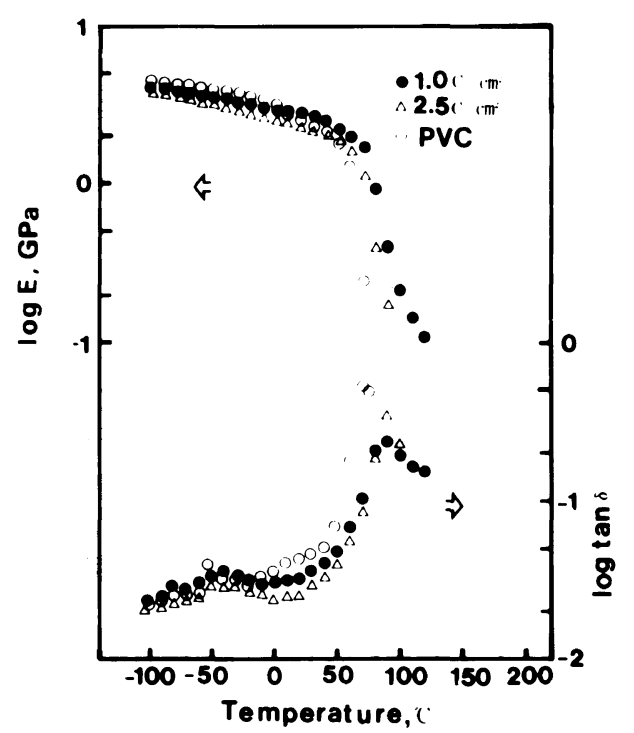

Figure 10. Temperature dependence of dynamic moduli of elasticity and loss moduli $(\tan \delta)$ for PVCPPy alloy films.

indicating that the crystallinity of P(VDFTrFE) film does not change only by immersing in the polymerization solution. Taking into account the reduction of $E$ during polymerization, the variation of crystalinity of $\mathrm{P}(\mathrm{VDF}-$ TrFE)-PPy may change by incorporating PPy. In the previous morphological study, it was reported that $\mathrm{P}(\mathrm{VDF}-\mathrm{TrFE})$ can be easily removed from the alloy films, and the fine network structure of PPy is observed by $\mathrm{SEM}^{9}$. This suggests that the phase separation structure of PPy and the base polymer exists in the alloy films, and this structure may attain good elongation of P(VDF-TrFE). However, $\tan \delta$ of $\mathrm{P}(\mathrm{VDF}-\mathrm{TrFE})-\mathrm{PPy}$ has a clear dependence on charge density. The maximum peak of $\tan \delta$ at $-38^{\circ} \mathrm{C}$ of P(VDF-TrFE)-PPy alloy films due to $T_{\mathrm{g}}$ of the base polymer becomes broader by increasing PPy content, and almost disappears at a charge density of $2.5 \mathrm{C} \mathrm{cm}^{-2}$.

This large variation of $\tan \delta$ peak due to $T_{\mathrm{g}}$ indicates that the phase separation of PPy and $\mathrm{P}(\mathrm{VDF}-\mathrm{TrFE})$ is not complete, and several fractions of PPy and P(VDF-TrFE) chains are in the miscible state. The molecular motion of $\mathrm{P}(\mathrm{VDF}-\mathrm{TrFE})$ chains in the miscible state is suppressed by the incorporation of rigid PPy chains.

Figure 10 shows the same results for PVCPPy films. $E$ of PVC-PPy does not vary by PPy incorporation. The peak temperature of $\tan \delta$ near $80^{\circ} \mathrm{C}$ due to $T_{\mathrm{g}}$ of PVC shifts a little to a higher temperature and its magnitude slightly reduces by alloying PPy. These results show that the suppression of the molecular motion of PVC by alloying PPy is not so large, and the phase separation between PVC and PPy is larger than in the case between $P(V D F-T r F E)$ and PPy. The better mechanical properties of PVC-PPy compared with P(EVA-VC)-PPy may be due to the large phase separation structure of PVC-PPy films.

\section{CONCLUSIONS}

Conducting PPy based polymer alloy films were prepared on an electrode coated with insulating polymer films by the electrochemical polymerization of pyrrole. By using three different types of insulating polymer films, the mechanical properties of conducting polymer alloy films were studied. P(VDF-TrFE), a crystalline and very low $T_{\mathrm{g}}$ polymer, gave a very flexible conducting film, and was stretchable by $120 \%$. No significant loss of conductivity was observed by $50 \%$ elongation. The mechanical properties of P(VDF-TrFE)PPy showed gradual deterioration by the incorporaion of PPy, probably because the fine mixing of PPy destroys the continuous P(VDF-TrFE) structure. PVC, an almost amorphous and high $T_{\mathrm{g}}$ polymer, and $\mathrm{P}(\mathrm{EVA}-$ VC), an amorphous and low $T_{\mathrm{g}}$ polymer, also gave conductive polymer films which also improved the low ductility of PPy by inheriting the mechanical properties of each base film. These films showed lower $\gamma_{\mathbf{B}}$ of elongation with breakage perhaps due to the high $T_{\mathrm{g}}$ of base polymer (PVC) or low capability in the rearrangement and orientation of the main 
polymer chains under stress $[\mathrm{P}(\mathrm{EVA}-\mathrm{VC})]$.

\section{REFERENCES}

1. J. Bargon, S. Mohmand, and R. J. Waltman, IBM J. Res. Develop., 27, 330 (1983).

2. A. F. Diaz and K. K. Kanazawa, Chem. Soc., Chem. Commun., 635 (1979).

3. A. F. Diaz and B. Hall, IBM J. Res. Develop., 27, 342 (1983).

4. M. Ogasawara, K. Funahashi, and K. Iwata, Mol. Cryst. Liq. Cryst., 118, 159 (1985).

5. N. Bates, M. Cross, R. Lines, and D. Walton, J.
Chem. Soc., Chem. Commun., 871 (1985).

6. O. Niwa and T. Tamamura, J. Chem. Soc., Chem. Commun., 817 (1984).

7. M. De Paoli, R. J. Waltman, A. F. Diaz, and J. Bargon, J. Chem. Soc., Chem. Commun., 1015 (1984).

8. O. Niwa, M. Hikita, and T. Tamamura, Macromol. Chem., Rapid Commun., 6, 375 (1985).

9. O. Niwa, M. Kakuchi, and T. Tamamura, Macromolecules, in press.

10. O. Niwa, M. Hikita, and T. Tamamura, Appl. Phys. Lett., 46, 444 (1985).

11. M. De Paoli, R. J. Waltman, A. F. Diaz, and J. Bargon, J. Polym. Sci., Polym. Chem. Ed., 23, 1687 (1985). 